\title{
Ground state solutions and infinitely many solutions for a nonlinear Choquard equation
}

Tianfang Wang ${ }^{1}$ and Wen Zhang ${ }^{2,3,4^{*}}$

${ }^{\text {*Correspondence: }}$

zwmath2011@163.com

${ }^{2}$ College of Science, Hunan University of Technology and

Business, Changsha, 410205, Hunan, P.R. China

${ }^{3}$ Key Laboratory of Hunan Province for Statistical Learning and Intelligent Computation, Hunan University of Technology and Business, Changsha, 410205, Hunan, P.R. China

Full list of author information is available at the end of the article

\section{Abstract}

In this paper we study the existence and multiplicity of solutions for the following nonlinear Choquard equation:

$$
-\Delta u+v(x) u=\left[|x|^{-\mu} *|u|^{p}\right]|u|^{p-2} u, \quad x \in \mathbb{R}^{N}
$$

where $N \geq 3,0<\mu<N, \frac{2 N-\mu}{N} \leq p<\frac{2 N-\mu}{N-2}$, * represents the convolution between two functions. We assume that the potential function $V(x)$ satisfies general periodic condition. Moreover, by using variational tools from the Nehari manifold method developed by Szulkin and Weth, we obtain the existence results of ground state solutions and infinitely many pairs of geometrically distinct solutions for the above problem.

MSC: $35 J 20 ; 35 J 70 ; 35 P 05$

Keywords: Choquard equation; Ground state solutions; Geometrically distinct solutions

\section{Introduction and main result}

Consider the following Choquard equation:

$$
-\Delta u+V(x) u=\left[|x|^{-\mu} *|u|^{p}\right]|u|^{p-2} u, \quad x \in \mathbb{R}^{N},
$$

where $N \geq 3,0<\mu<N, \frac{2 N-\mu}{N} \leq p<\frac{2 N-\mu}{N-2}$. Problem (1.1) arises from the study of the existence of standing wave solutions for the following equation:

$$
i \psi_{t}=-\Delta \psi+W \psi-\left[|x|^{-\mu} *|\psi|^{p}\right]|\psi|^{p-2} \psi
$$

which appears naturally in optical systems with a thermal [21] and influences the propagation of electromagnetic waves in plasmas [2] and plays an important role in the theory of Bose-Einstein condensation [9]. Here $\psi: \mathbb{R}^{N} \times \mathbb{R} \rightarrow \mathbb{C}$ represents the wave function of the state of an electron, and $W$ is the external potential. In the present paper, we are mainly interested in studying the standing wave solutions of the form $\psi(x, t)=u(x) e^{-i E t}$. This type of particle-like solution does not change its shape as it evolves in time, hence

(c) The Author(s) 2021. This article is licensed under a Creative Commons Attribution 4.0 International License, which permits use, sharing, adaptation, distribution and reproduction in any medium or format, as long as you give appropriate credit to the original author(s) and the source, provide a link to the Creative Commons licence, and indicate if changes were made. The images or other third party material in this article are included in the article's Creative Commons licence, unless indicated otherwise in a credit line 
has a soliton-like behavior. Obviously, $u(x)$ solves (1.1) iff $\psi(x, t)$ solves the above equation with $V(x)=W(x)-E$. Additionally, it is easy to see that problem (1.1) has nonlocal characteristics in the nonlinearity due to the effect of the convolution part, which is different from a local problem.

Especially, when $p=2, N=3$, and $\mu=1$, problem (1.1) is called Choquard-Pekar equation

$$
-\Delta u+V(x) u=\left[|x|^{-1} *|u|^{2}\right] u, \quad x \in \mathbb{R}^{3},
$$

which arises in the description of the quantum theory of a polaron at rest by Peak [28] in 1954 and in the modeling of an electron trapped in its own hole in 1976 in the work of Choquard, see [18]. Moreover, people also call this equation the Schrödinger-Newton equation, which was introduced by Penrose in his discussion on the self-gravitational collapse of a quantum mechanical wave function [29]. Up to reparametrization, Penrose suggested that the solutions to (1.2) are the basic stationary states that do not spontaneously collapse any further within a certain time scale. Hence, it is very interesting to study these basic solutions. Besides, equation (1.2) has many interesting applications in the quantum theory of large systems of nonrelativistic bosonic atoms and molecules, we refer readers to $[18,28]$ for more physical backgrounds.

Mathematical work on nonlinear Choquard equations like the above has been investigated in recent years, and the existence and multiplicity results for such type equations have been considered in many papers under some different assumptions on the potential and nonlinearity by using various variational arguments. For readers' convenience, next we briefly summarize the related study on the existence and multiplicity of nontrivial solutions to problem (1.1).

We pointed out that Lieb [18] and Lions [20] firstly studied the existence and symmetry of the solutions to (1.2). More precisely, up to translations, Lieb [18] obtained the existence and uniqueness of the ground state solutions with $V$ being a positive constant. Lions [20] showed the existence of a sequence of radially symmetric solutions. Since then people began to pay attention to studying the existence of nontrivial solutions for nonlinear Choquard equations, not only from the mathematical curiosity. Such nonlocal problems are also widely used in optimization, finance, phase transitions, stratified materials, anomalous diffusion, and so on. Although Lieb [18] established the uniqueness of the ground state solutions, the classification of positive solutions has been an open problem for many years. The fundamental reason is that people cannot use the standard method of moving planes (based on the maximum principle) to obtain the radial symmetry of the solutions. Until 2010, inspired by the works of Chen et al. [4] and Li et al. [17], under the assumptions $p \geq 2$ and

$$
\left[2, \frac{2 N}{N-2}\right] \cap\left(p, \frac{N p}{\mu}\right) \cap\left(\frac{(2 p-2) N}{\mu+2}, \frac{(2 p-1) N}{\mu+2}\right) \cap\left[\frac{(2 p-1) N}{N+\mu},+\infty\right) \neq \emptyset,
$$

Ma and Zhao [22] proved that all the positive solutions to equation (1.1) with $V=$ Const and $2 \leq p<\frac{2 N-\mu}{N-2}$ are radially symmetry and monotone decreasing about some fixed point. And by using of the new method of moving planes introduced in [4] and Riesz and Bessel potentials, they deduced the problem into an elliptic system. At last, using the radial symmetry, up to translations, they proved that the positive solution to (1.2) (not only the 
ground state) is unique, which solved the open problem in [18]. After that, in the spirit of [22], Cingolani, Clapp, and Secchi [5] considered the following nonlinear Choquard equation with magnetic field:

$$
\left\{\begin{array}{l}
(-i \nabla+A(x))^{2} u+V(x) u=\left[|x|^{-\mu} *|u|^{p}\right]|u|^{p-2} u, \quad x \in \mathbb{R}^{N}, \\
u \in L^{2}\left(\mathbb{R}^{N}, \mathbb{C}\right) \\
\nabla u+i A(x) u \in L^{2}\left(\mathbb{R}^{N}, \mathbb{C}\right)
\end{array}\right.
$$

and obtained the existence of multiple complex-valued solutions that satisfy the symmetry condition $u(g x)=\tau(g) u(x)$ for all $g \in G$, where $\tau: G \rightarrow S_{1}$ is a given group homomorphism into the unit complex numbers, $A$ is a real-valued $C^{1}$-vector potential, $V$ is a real-valued bounded continuous scalar potential with $\inf V>0, N \geq 3,0<\mu<N$, and $\frac{2 N-\mu}{N}<p<\frac{2 N-\mu}{N-2}$. In [24], Moroz and Van Schaftingen eliminated this restriction (1.3) to establish regularity and positivity of the groundstates for equation (1.1). They also proved the radial symmetry and monotonic decay of positive groundstates. We pointed out that they had many interesting works about Choquard equation, see [25-27] and the references therein. Recently, the strongly indefinite Choquard equations with critical exponent in the whole space were also studied in $[14,31,32,39]$ where the existence and multiplicity were obtained by using a linking theorem. Furthermore, by using the minimax procedure and perturbation technique, Gao et al. [15] showed the existence of infinitely many solutions for a class of critical Choquard equations with zero mass.

Very recently, for other related topics involving the singularly perturbed problem, there have been some works devoted to the study of a concentration phenomenon of semiclassical states. For instance, by using a Lyapunov-Schmidt type reduction, Wei and Winter [37] constructed families of solutions for the following equation:

$$
-\varepsilon^{2} \Delta u+V(x) u=\varepsilon^{-2}\left[|x|^{-1} *|u|^{2}\right] u, \quad x \in \mathbb{R}^{3}
$$

with potential $\inf V>0$ and characterized the concentration behavior around the global minimum points of $V$. Moreover, they also showed that the groundstate to (1.2) is up to translations a nondegenerate critical point. Not long after that the existence of a family of solutions having multiple concentration regions located around the minimum points of the potential was obtained in [6]. With the help of the mountain pass lemma and the genus theory, Ding et al. [10] obtained the existence and multiplicity of semiclassical states to the Choquard equation

$$
-\varepsilon^{2} \Delta u+V(x) u=\left(\int_{\mathbb{R}^{N}} \frac{G(u(y))}{|x-y|^{\mu}} d y\right) g(u) .
$$

They also constructed the multiplicity of high energy semiclassical states by using the Ljusternik-Schnirelmann theory. In [42], the authors proved the existence and concentration of semiclassical solutions under Berestycki-Lions type conditions. Furthermore, the other type of Choquard equations has also attracted great interest. For example, instead of the classical Laplacian operator, many scholars considered the following type of Choquard equation:

$$
(-\Delta)^{s} u+V(x) u=\left[|x|^{-\mu} * F(u)\right] f(u), \quad x \in \mathbb{R}^{N},
$$


which is called fractional Choquard equation that is used to model the dynamics of pseudo-relativistic boson stars. About the study of fractional Choquard equations, please see $[8,23,34]$ and their references therein. More related results about ground state solutions and infinitely many solutions for other problems, we refer the readers to $[1,3,7,13$, $16,30,33,41,43,44]$ and the references therein.

Motivated by the results mentioned above, in this paper we consider the case that the potential $V$ is a general periodic function and prove the existence of a ground state solution and infinitely many pairs of geometrically distinct solutions for problem (1.1) by using the method of the Nehari manifold developed by Szulkin and Weth [36]. To the best of our knowledge, it seems that there is no work that considered this problem in the literature before. In order to state the main result, we list the assumption as follows:

$(V) V \in C\left(\mathbb{R}^{N}, \mathbb{R}\right), 0<V_{0}:=\inf _{x \in \mathbb{R}^{N}} V(x)$ and $V(x)$ is 1-periodic in $x_{i}, i=1, \ldots, N$.

Set

$$
E=\left\{u \in H^{1}\left(\mathbb{R}^{N}\right): \int_{\mathbb{R}^{N}}\left[|\nabla u|^{2}+V(x) u^{2}\right] d x<+\infty\right\}
$$

with the norm

$$
\|u\|^{2}=\int_{\mathbb{R}^{N}}\left[|\nabla u|^{2}+V(x) u^{2}\right] d x
$$

Then, according to condition $(V)$, the norm $\|\cdot\|$ is equivalent to the norm $\|\cdot\|_{H^{1}}$, where

$$
\|u\|_{H^{1}}^{2}=\int_{\mathbb{R}^{N}}\left[|\nabla u|^{2}+u^{2}\right] d x .
$$

For $k \in \mathbb{Z}^{N}$, set $(k * u)(x)=u(x+k)$. According to the periodicity condition $(V)$, if $u_{0}$ is a solution of (1.1), then $k * u_{0}$ is also a solution of (1.1) for all $k \in \mathbb{Z}^{N}$. Set

$$
\mathcal{O}\left(u_{0}\right):=\left\{k * u_{0}: k \in \mathbb{Z}^{N}\right\}
$$

which means the orbit of $u_{0}$ with respect to the action of $\mathbb{Z}^{N}$. If $u_{0}$ is a critical point of the energy functional $J$ and $J$ is $\mathbb{Z}^{N}$-invariant, i.e., $J(k * u)=J(u)$ for all $k \in \mathbb{Z}^{N}$ and all $u \in E$, we call $\mathcal{O}\left(u_{0}\right)$ a critical orbit of $J$. Suppose that $u_{i}(i=1,2)$ solves $(1.1)$, if $\mathcal{O}\left(u_{1}\right) \neq \mathcal{O}\left(u_{2}\right)$, we say that $u_{1}, u_{2}$ are geometrically distinct.

Our main result of this paper is the following:

Theorem 1.1 Assume that ( $V$ ) holds. Then equation (1.1) admits a ground state solution and infinitely many pairs of geometrically distinct solutions.

\section{Proof of theorem}

Throughout this paper, we denote by $\|\cdot\|_{s}$ the usual norm of the space $L^{s}, 1 \leq s \leq \infty$, and $c$ or $c_{i}(i=1,2, \ldots)$ denotes the different positive constants.

Firstly, in order to overcome the nonlocality of problem (1.1) and study the property of the energy functional, we will use the classical Hardy-Littlewood-Sobolev inequality frequently. So, we give the Hardy-Littlewood-Sobolev inequality due to [19]. 
Proposition 2.1 (Hardy-Littlewood-Sobolev inequality, [19]) Let $s, t>1$ and $0<\mu<N$ with $\frac{1}{s}+\frac{\mu}{N}+\frac{1}{t}=2$. Let $g \in L^{s}\left(\mathbb{R}^{N}\right)$ and $h \in L^{t}\left(\mathbb{R}^{N}\right)$. Then there exists a sharp constant $C_{s, N, \mu, t}$, independent of $g, h$, such that

$$
\int_{\mathbb{R}^{N}} \int_{\mathbb{R}^{N}} \frac{g(x) h(y)}{|x-y|^{\mu}} d x d y \leq C_{s, N, \mu, t}|g|_{s}|h|_{t} .
$$

Remark 2.2 Obviously, Hardy-Littlewood-Sobolev inequality implies that the integral

$$
\int_{\mathbb{R}^{N}} \int_{\mathbb{R}^{N}} \frac{|u(x)|^{q}|u(y)|^{q}}{|x-y|^{\mu}} d x d y
$$

is well defined if $|u|^{q} \in L^{t}\left(\mathbb{R}^{N}\right)$ for $t>1$ with $\frac{2}{t}+\frac{\mu}{N}=2$. Since we will work with $u \in E$, in order to make the integral well defined, $t q$ must fall in the interval $\left[2,2^{*}\right]$, i.e.,

$$
\frac{2 N-\mu}{N} \leq q \leq \frac{2 N-\mu}{N-2}
$$

That is why the exponent $\frac{2 N-\mu}{N}$ is called the lower critical exponent and the exponent $\frac{2 N-\mu}{N-2}$ is called the upper critical exponent. We need to point out that Hardy-LittlewoodSobolev inequality plays an important role for nonlocal problems.

From a viewpoint of variational methods, it is clear that equation (1.1) is the EulerLagrange equation associated with the energy functional $J: E \rightarrow \mathbb{R}$ given by

$$
J(u)=\frac{1}{2} \int_{\mathbb{R}^{N}}\left[|\nabla u|^{2}+V(x)|u|^{2}\right] d x-\frac{1}{2 p} \int_{\mathbb{R}^{N}}\left[|x|^{-\mu} *|u|^{p}\right]|u|^{p} d x .
$$

Since $p \in\left[\frac{2 N-\mu}{N}, \frac{2 N-\mu}{N-2}\right)$, then by Hardy-Littlewood-Sobolev inequality and Sobolev embedding theorem, it is easy to prove that $J$ is well defined on $E$ and belongs to $C^{1}(E, \mathbb{R})$. Moreover, there holds

$$
\left\langle J^{\prime}(u), \varphi\right\rangle=\int_{\mathbb{R}^{N}}[\nabla u \cdot \nabla \varphi+V(x) u \varphi] d x-\int_{\mathbb{R}^{N}}\left[|x|^{-\mu} *|u|^{p}\right]|u|^{p-2} u \varphi d x
$$

for all $u, \varphi \in E$.

In order to seek for the ground state solutions of problem (1.1), we consider the following Nehari set:

$$
\mathcal{N}=\left\{u \in E \backslash\{0\}:\left\langle J^{\prime}(u), u\right\rangle=0\right\}
$$

and define the ground state energy

$$
c:=\inf _{u \in \mathcal{N}} J(u)
$$

Lemma 2.3 For $s \geq 0$, let $f(s)=J(s v)$. Then, for each $v \in E \backslash\{0\}$, there exists unique $t_{v}>0$ such that $f\left(t_{v}\right)=\max _{s \geq 0} f(s), f^{\prime}(t)>0$ for $0<t<t_{v}$ and $f^{\prime}(t)<0$ for $t_{v}<t$. Moreover, $t v \in \mathcal{N}$ if and only if $t=t_{\nu}$. 
Proof According to Hardy-Littlewood-Sobolev inequality and Sobolev embedding theorem, we have

$$
\begin{aligned}
f(t) & =J(t v)=\frac{1}{2} t^{2}\|v\|^{2}-\frac{1}{2 p} t^{2 p} \int_{\mathbb{R}^{N}}\left[|x|^{-\mu} *|v|^{p}\right]|v|^{p} d x \\
& \geq \frac{1}{2} t^{2}\|v\|^{2}-\frac{1}{2 p} t^{2 p}\|v\|^{2 p}>0
\end{aligned}
$$

for small $t>0$. Clearly,

$$
f(t)=J(t v) \rightarrow-\infty
$$

as $t \rightarrow+\infty$. Hence $f$ has a positive maximum and there exists $t_{v}>0$ such that $f^{\prime}\left(t_{v}\right)=0$.

Consequently,

$$
\|v\|^{2}=t_{v}^{2 p-2} \int_{\mathbb{R}^{N}}\left[|x|^{-\mu} *|v|^{p}\right]|v|^{p} d x
$$

from where it follows that $f^{\prime}(t)>0$ for $0<t<t_{v}$ and $f^{\prime}(t)<0$ for $t_{v}<t$ and $t_{v}$ is unique. The proof is completed.

For the sake of convenience, for $r>0$, set $B_{r}:=\{v \in E:\|v\| \leq r\}$ and $S_{r}:=\{v \in E:\|v\|=r\}$.

Lemma 2.4 (i) There are constants $\varrho>0$ and $\alpha>0$ such that $c \geq \inf _{S_{\varrho}} J \geq \alpha>0$;

(ii) $\|v\| \geq \sqrt{2 c}>0$ for all $v \in \mathcal{N}$.

Proof (i) Let $v \in E$, by the Hardy-Littlewood-Sobolev inequality and the Sobolev embedding theorem, we get

$$
\begin{aligned}
J(v) & =\frac{1}{2}\|v\|^{2}-\frac{1}{2 p} \int_{\mathbb{R}^{N}}\left[|x|^{-\mu} *|v|^{p}\right]|v|^{p} d x \\
& \geq \frac{1}{2}\|v\|^{2}-\frac{1}{2 p} c_{1}\|v\|^{2 p} .
\end{aligned}
$$

Observe that $0<\mu<N$, then $p>1$. So, from the above fact, it is easy to see that there exist $\varrho>0$ and $\alpha>0$ such that $\inf _{S_{\varrho}} J \geq \alpha>0$. On the other hand, for every $v \in \mathcal{N}$, there exists $t_{0}>0$ such that $t_{0} v \in S_{\varrho}$. Then by Lemma 2.3 we get

$$
\inf _{S_{\varrho}} J \leq J\left(t_{0} v\right) \leq \max _{t>0} J(t v)=J(v)
$$

which yields that $c \geq \inf _{S_{e}} J \geq \alpha>0$.

(ii) For $v \in \mathcal{N}$, there holds

$$
c=\inf _{\mathcal{N}} J \leq J(v)=\frac{1}{2}\|v\|^{2}-\frac{1}{2 p} \int_{\mathbb{R}^{N}}\left[|x|^{-\mu} *|v|^{p}\right]|v|^{p} d x \leq \frac{1}{2}\|v\|^{2},
$$

which implies that $\|v\| \geq \sqrt{2 c}>0$ for all $v \in \mathcal{N}$. The proof is completed.

Lemma 2.5 $J$ is coercive on $\mathcal{N}$, i.e., $J(v) \rightarrow+\infty$ as $v \in \mathcal{N}$ and $\|v\| \rightarrow \infty$. 
Proof Arguing by contradiction, we assume that there exist a sequence $\left\{v_{n}\right\} \subset \mathcal{N}$ and a positive number $m$ such that $\left\|v_{n}\right\| \rightarrow \infty$ and $J\left(v_{n}\right) \leq m$. Set $u_{n}=\frac{v_{n}}{\left\|v_{n}\right\|}$. Then, passing to a subsequence, there exists $u \in E$ such that $u_{n} \rightarrow u$ in $E, u_{n} \rightarrow u$ in $L_{l o c}^{q}\left(\mathbb{R}^{N}\right)$ for any $2 \leq q<2^{*}$ and $u_{n}(x) \rightarrow u(x)$ a.e. on $\mathbb{R}^{N}$. By the fact that $u_{n} \neq 0$, there exists a point $y \in \mathbb{R}^{N}$ such that

$$
\int_{B_{1}(y)} u_{n}^{2} d x:=\delta>0
$$

Set

$$
\gamma(z):=\int_{B_{1}(z)} u_{n}^{2} d x
$$

It is easy to prove that $\gamma(z)$ is continuous on $\mathbb{R}^{N}$ by the absolute continuity of integral. Take large $R>0$ with

$$
\int_{\mathbb{R}^{N} \backslash B_{R}(0)} u_{n}^{2} d x<\delta
$$

Then

$$
\gamma(z)=\int_{B_{1}(z)} u_{n}^{2} d x<\delta
$$

for any $z \in \mathbb{R}^{N} \backslash B_{R+1}(0)$. Consequently,

$$
\sup _{z \in \mathbb{R}^{N}} \gamma(z)=\sup _{z \in \bar{B}_{R+1}(0)} \gamma(z)
$$

Note that $\gamma$ is continuous and $\bar{B}_{R+1}(0)$ is a compact set, there exists $y_{n} \in \bar{B}_{R+1}(0)$ such that $\gamma\left(y_{n}\right)=\sup _{z \in \bar{B}_{R+1}(0)} \gamma(z)$. Hence,

$$
\int_{B_{1}\left(y_{n}\right)} u_{n}^{2} d x=\sup _{z \in \mathbb{R}^{N}} \int_{B_{1}(z)} u_{n}^{2} d x
$$

According to the assumption of periodicity, we can assume that $\left\{y_{n}\right\}$ is bounded in $\mathbb{R}^{N}$. In the sequel, we prove

$$
\limsup _{n \rightarrow \infty} \int_{B_{1}\left(y_{n}\right)} u_{n}^{2} d x>0
$$

Indeed, if

$$
\lim _{n \rightarrow \infty} \int_{B_{1}\left(y_{n}\right)} u_{n}^{2} d x=0
$$

using the vanishing lemma [38, Lemma 1.21], we have $u_{n} \rightarrow 0$ in $L^{s}\left(\mathbb{R}^{N}\right)$ for $2<s<2^{*}$. Hence, for any $t>0$, there holds

$$
\begin{aligned}
m & \geq J\left(v_{n}\right)=\max _{s \geq 0} J\left(s v_{n}\right) \geq J\left(\frac{t}{\left\|v_{n}\right\|} v_{n}\right)=J\left(t u_{n}\right) \\
& =\frac{t^{2}}{2}-\frac{1}{2 p} t^{2 p} \int_{\mathbb{R}^{N}}\left[|x|^{-\mu} *\left|u_{n}\right|^{p}\right]\left|u_{n}\right|^{p} d x \rightarrow \frac{t^{2}}{2},
\end{aligned}
$$


this is impossible if $t$ is large enough. Therefore, we know that $u \neq 0$. Set $\Omega=\left\{x \in \mathbb{R}^{N}\right.$ : $u(x) \neq 0\}$, then meas $(\Omega)>0$. In the light of Fatou's lemma we deduce that

$$
\begin{aligned}
& \liminf _{n \rightarrow \infty} \int_{\mathbb{R}^{N}} \frac{\left[|x|^{-\mu} *\left|v_{n}\right|^{p}\right]\left|v_{n}\right|^{p}}{\left\|v_{n}\right\|^{2}} d x \\
& \quad=\liminf _{n \rightarrow \infty}\left(\left\|v_{n}\right\|_{E}^{2 p-2} \int_{\mathbb{R}^{N}}\left[|x|^{-\mu} *\left|u_{n}\right|^{p}\right]\left|u_{n}\right|^{p} d x\right) \\
& \quad \geq \int_{\Omega} \int_{\Omega} \frac{|u(x)|^{p}|u(y)|^{p}}{|x-y|^{\mu}} d x d y \cdot \liminf _{n \rightarrow \infty}\left\|v_{n}\right\|^{2 p-2}=+\infty .
\end{aligned}
$$

Then it follows that

$$
0 \leq \frac{J\left(v_{n}\right)}{\left\|v_{n}\right\|^{2}}=\frac{1}{2}-\frac{1}{2 p} \int_{\mathbb{R}^{N}} \frac{\left[|x|^{-\mu} *\left|v_{n}\right|^{p}\right]\left|v_{n}\right|^{p}}{\left\|v_{n}\right\|^{2}} d x \rightarrow-\infty
$$

as $n \rightarrow \infty$, which yields a contradiction. The proof is completed.

Let $\mathcal{W} \subset E \backslash\{0\}$ be a compact subset. Set $\mathbb{R}^{+} \mathcal{W}:=\left\{t v: t \in \mathbb{R}^{+}, v \in \mathcal{W}\right\}$.

Lemma 2.6 There exists $r>0$ such that $J(v)<0$ on $\left(\mathbb{R}^{+} \mathcal{W}\right) \backslash B_{r}$ for each $v \in \mathcal{W}$.

Proof Without loss of generality, we may assume that $\|v\|=1$ for every $v \in \mathcal{W}$. Suppose that there exist $v_{n} \in \mathcal{W}$ and $w_{n}=t_{n} v_{n}$ such that $J\left(w_{n}\right) \geq 0$ and $t_{n} \rightarrow \infty$ as $n \rightarrow \infty$. Passing to a subsequence, we may assume that $v_{n} \rightarrow v \in S_{1}$ in $\mathcal{W} \subset E \backslash\{0\}$. Consequently, using Fatou's lemma, we obtain

$$
\begin{aligned}
0 & \leq J\left(w_{n}\right)=J\left(t_{n} v_{n}\right)=\frac{1}{2} t_{n}^{2}\left\|v_{n}\right\|_{E}^{2}-\frac{1}{2 p} t_{n}^{2 p} \int_{\mathbb{R}^{N}}\left[|x|^{-\mu} *\left|v_{n}\right|^{p}\right]\left|v_{n}\right|^{p} d x \\
& =t_{n}^{2}\left(\frac{1}{2}-\frac{1}{2 p} t_{n}^{2 p-2} \int_{\mathbb{R}^{N}}\left[|x|^{-\mu} *\left|v_{n}\right|^{p}\right]\left|v_{n}\right|^{p} d x\right) \\
& \rightarrow-\infty
\end{aligned}
$$

as $n \rightarrow \infty$, a contradiction. This completes the proof.

For convenience, set $S_{1}=S$. Introduce the mapping $\tilde{m}: E \backslash\{0\} \rightarrow \mathcal{N}$ and $m: S \rightarrow \mathcal{N}$ by setting

$$
\tilde{m}(v)=t_{v} v \quad \text { and } \quad m=\left.\tilde{m}\right|_{S},
$$

where $t_{v}$ comes from Lemma 2.3. Set

$$
\tilde{\psi}(v)=J(\tilde{m}(v)) \text { and } \quad \psi=\left.\tilde{\psi}\right|_{s} .
$$

Since $E$ is a Hilbert space, by Lemmas $2.3,2.4$, and 2.6 we can verify that the hypotheses $A_{2}$ and $A_{3}$ in [36] hold. Hence, we have Lemmas 2.7-2.8, the details of proofs can be found in [36].

Lemma 2.7 ([36, Proposition 3.1]) The mapping $\tilde{m}: E \backslash\{0\} \rightarrow \mathcal{N}$ is continuous and $m$ is a homeomorphism between $S$ and $\mathcal{N}$, and the inverse of $m$ is given by $m^{-1}(v)=\frac{v}{\|v\|}$. 
Lemma 2.8 ([36, Corollary 3.3])

(i) $\psi \in C^{1}(S, \mathbb{R})$ and

$$
\psi^{\prime}(w) z=\|m(w)\| J^{\prime}(m(w)) z
$$

for all $z \in T_{w}(S):=\{v \in E:\langle w, v\rangle=0\}$.

(ii) If $\left\{w_{n}\right\}$ is a Palais-Smale sequence for $\psi$, then $\left\{m\left(w_{n}\right)\right\}$ is a Palais-Smale sequence for $J$. If $\left\{v_{n}\right\} \subset \mathcal{N}$ is a bounded Palais-Smale sequence for $J$, then $\left\{m^{-1}\left(v_{n}\right)\right\}$ is a Palais-Smale sequence for $\psi$.

(iii) $w \in S$ is a critical point of $\psi$ iff $m(w)$ is a nontrivial critical point of J. Moreover, the corresponding values of $\psi$ and $J$ coincide and $\inf _{S} \psi=\inf _{\mathcal{N}} J$.

(iv) IfJ is even, then so is $\psi$.

Lemma 2.9 The mapping $m^{-1}$ defined in Lemma 2.7 is Lipschitz continuous.

Proof For any $v, w \in \mathcal{N}$, by Lemma 2.4-(ii) we have $\|v\| \geq \sqrt{2 c}$ and $\|w\| \geq \sqrt{2 c}$. Moreover, using Lemma 2.7, we obtain

$$
\begin{aligned}
\left\|m^{-1}(v)-m^{-1}(w)\right\| & =\left\|\frac{v}{\|v\|}-\frac{w}{\|w\|}\right\|=\left\|\frac{v-w}{\|v\|}+\frac{(\|w\|-\|v\|) w}{\|v\|\|w\|}\right\| \\
& \leq \frac{2}{\|v\|}\|v-w\| \leq \sqrt{\frac{2}{c}}\|v-w\| .
\end{aligned}
$$

From the above fact, it is easy to see that the mapping $m^{-1}$ is Lipschitz continuous. This completes the proof.

For any $a \in \mathbb{R}$, set $\psi^{a}:=\{v \in S: \psi(v) \leq a\}$. We have the following lemma about the discreteness property of the Palais-Smale sequence.

Lemma 2.10 Let $d \geq c$. If $\left\{v_{n}^{1}\right\},\left\{v_{n}^{2}\right\} \subset \psi^{d}$ are two Palais-Smale sequences of $\psi$, then one of the following conclusions holds:

(i) $\left\|v_{n}^{1}-v_{n}^{2}\right\| \rightarrow 0$ as $n \rightarrow \infty$;

(ii) $\liminf _{n \rightarrow \infty}\left\|v_{n}^{1}-v_{n}^{2}\right\| \geq \rho(d)>0$, where $\rho(d)$ is a constant that depends on $d$ but not on the particular choice of Palais-Smale sequences.

Proof Set $u_{n}^{1}:=m\left(v_{n}^{1}\right)$ and $u_{n}^{2}:=m\left(v_{n}^{2}\right)$. By Lemma 2.8-(ii), $\left\{u_{n}^{i}\right\}(i=1,2)$ is a PalaisSmale sequence of $J$ and $\left\{u_{n}^{1}\right\},\left\{u_{n}^{2}\right\} \subset J^{d}$. Then $\left\{u_{n}^{1}\right\},\left\{u_{n}^{2}\right\}$ are bounded in $E$ according to Lemma 2.5. Computing directly, we have

$$
\begin{aligned}
o(1)= & \left\langle J^{\prime}\left(u_{n}^{1}\right)-J^{\prime}\left(u_{n}^{2}\right), u_{n}^{1}-u_{n}^{2}\right\rangle \\
= & \left\|u_{n}^{1}-u_{n}^{2}\right\|^{2}-\int_{\mathbb{R}^{N}}\left(\left[|x|^{-\mu} *\left|u_{n}^{1}\right|^{p}\right]\left|u_{n}^{1}\right|^{p-2} u_{n}^{1}\right. \\
& \left.-\left[|x|^{-\mu} *\left|u_{n}^{2}\right|^{p}\right]\left|u_{n}^{2}\right|^{p-2} u_{n}^{2}\right)\left(u_{n}^{1}-u_{n}^{2}\right) d x .
\end{aligned}
$$

Consequently, by the Hardy-Littlewood-Sobolev inequality, Hölder's inequality, and the boundedness of $\left\{u_{n}^{1}\right\}$ and $\left\{u_{n}^{2}\right\}$ in $E$, we know that there exists a constant $c_{2}>0$ such that

$$
\left\|u_{n}^{1}-u_{n}^{2}\right\|^{2} \leq c_{2}\left\|u_{n}^{1}-u_{n}^{2}\right\|_{\frac{2 N p}{2 N-\mu}}^{2}+o(1) .
$$


In the following we divide into two cases to discuss.

Case 1: $\left\|u_{n}^{1}-u_{n}^{2}\right\|_{\frac{2 N p}{2 N-\mu}} \rightarrow 0$ as $n \rightarrow \infty$. It follows from (2.1) that $\left\|u_{n}^{1}-u_{n}^{2}\right\| \rightarrow 0$. Moreover, from Lemma 2.9 we have $\left\|v_{n}^{1}-v_{n}^{2}\right\|=\left\|m^{-1}\left(u_{n}^{1}\right)-m^{-1}\left(u_{n}^{2}\right)\right\| \rightarrow 0$.

Case 2: $\left\|u_{n}^{1}-u_{n}^{2}\right\|_{\frac{2 N p}{2 N-\mu}} \nrightarrow 0$ as $n \rightarrow \infty$. By the boundedness of $\left\{u_{n}^{1}\right\}$ and $\left\{u_{n}^{2}\right\}$ in $E$ and the vanishing lemma [38, Lemma 1.21], we can deduce that

$$
\sup _{y \in \mathbb{R}^{N}} \int_{B_{1}(v)}\left|u_{n}^{1}-u_{n}^{2}\right|^{\frac{2 N p}{2 N-\mu}} d x \rightarrow 0
$$

Thereby, up to a subsequence, there exists $\varepsilon_{0}>0$ such that

$$
\sup _{y \in \mathbb{R}^{N}} \int_{B_{1}(y)}\left|u_{n}^{1}-u_{n}^{2}\right|^{\frac{2 N p}{2 N-\mu}} d x \geq \varepsilon_{0}, \quad \forall n \in \mathbb{N} .
$$

Following the proof of Lemma 2.5 , there exists $y_{n} \in \mathbb{R}^{N}$ such that

$$
\int_{B_{1}\left(y_{n}\right)}\left|u_{n}^{1}-u_{n}^{2}\right|^{\frac{2 N p}{2 N-\mu}} d x=\sup _{y \in \mathbb{R}^{N}} \int_{B_{1}(y)}\left|u_{n}^{1}-u_{n}^{2}\right|^{\frac{2 N p}{2 N-\mu}} d x \geq \varepsilon_{0}, \quad \forall n \in \mathbb{N} .
$$

Similar to [12], with the help of Lemma 2.4 and Lemma 2.5, we can prove that $\liminf _{n \rightarrow \infty}\left\|v_{n}^{1}-v_{n}^{2}\right\| \geq \rho(d)>0$, where $\rho(d)$ depends on $d$ but not on the particular choice of Palais-Smale sequences. The proof is completed.

To complete the proof of Theorem 1.1, we take the following two lemmas.

Lemma 2.11 Problem (1.1) has at least a ground state solution.

Proof Set

$$
\varphi(u)=\int_{\mathbb{R}^{N}}\left[|\nabla u|^{2}+V(x) u^{2}\right] d x-1, \quad \forall u \in E .
$$

Then $S=\{u \in E: \varphi(u)=0\}$. For $u \in S$, one has

$$
\left\langle\varphi^{\prime}(u), u\right\rangle=2\|u\|^{2}=2>0 .
$$

Making use of Proposition 9 in [36], we know that $\tilde{\psi}: E \backslash\{0\} \rightarrow \mathbb{R}$ is a class of $C^{1}$. Moreover, it is easy to see that

$$
\left\langle\tilde{\psi}^{\prime}(u), v\right\rangle=\frac{\|\tilde{m}(u)\|}{\|u\|}\left\langle J^{\prime}(\tilde{m}(u)), v\right\rangle, \quad \forall 0 \neq u, v \in E .
$$

According to Corollary 3.4 in [11], there exists a sequence $\left\{w_{n}\right\} \subset S$ such that $\psi\left(w_{n}\right) \rightarrow c$ and there exists $\alpha_{n} \in \mathbb{R}$ such that $\left\|\tilde{\psi}^{\prime}\left(w_{n}\right)-\alpha_{n} \varphi^{\prime}\left(w_{n}\right)\right\|_{E^{*}} \rightarrow 0$. Hence,

$$
\alpha_{n}=\frac{\left\langle\tilde{\psi}^{\prime}\left(w_{n}\right), \varphi^{\prime}\left(w_{n}\right)\right\rangle}{\left\|\varphi^{\prime}\left(w_{n}\right)\right\|_{E^{*}}^{2}}+o(1)
$$


Then

$$
\tilde{\psi}^{\prime}\left(w_{n}\right)-\frac{\left\langle\tilde{\psi}^{\prime}\left(w_{n}\right), \varphi^{\prime}\left(w_{n}\right)\right\rangle}{\left\|\varphi^{\prime}\left(w_{n}\right)\right\|_{E^{*}}^{2}} \varphi^{\prime}\left(w_{n}\right)=o(1), \quad \text { i.e., } \psi^{\prime}\left(w_{n}\right)=o(1) .
$$

Set $v_{n}=m\left(w_{n}\right) \in \mathcal{N}$ for all $n \in \mathbb{N}$. In view of Lemma 2.8-(ii) we have

$$
J\left(v_{n}\right) \rightarrow c \quad \text { and } \quad J^{\prime}\left(v_{n}\right) \rightarrow 0 \quad \text { in } E^{*} .
$$

Moreover, $\left\{v_{n}\right\}$ is bounded in $E$ by Lemma 2.5 . Consequently, up to a subsequence, there exists $v \in E$ such that $v_{n} \rightarrow v$ in $E, v_{n} \rightarrow v$ in $L_{l o c}^{q}\left(\mathbb{R}^{N}\right)$ for any $2 \leq q<2^{*}$ and $v_{n}(x) \rightarrow v(x)$ a.e. on $\mathbb{R}^{N}$. In what follows, we claim that $J^{\prime}(v)=0$. In fact, for any $\phi \in C_{0}^{\infty}\left(\mathbb{R}^{N}\right)$, there holds

$$
o(1)=\left\langle J^{\prime}\left(v_{n}\right), \phi\right\rangle=\int_{\mathbb{R}^{N}} \nabla v_{n} \nabla \phi d x+\int_{\mathbb{R}^{N}} V(x) v_{n} \phi d x-\int_{\mathbb{R}^{N}}\left[|x|^{-\mu} *\left|v_{n}\right|^{p}\right]\left|v_{n}\right|^{p-2} v_{n} \phi d x .
$$

According to the fact that $v_{n} \rightarrow v$ in $E$, we get

$$
\int_{\mathbb{R}^{N}} \nabla v_{n} \nabla \phi d x \rightarrow \int_{\mathbb{R}^{N}} \nabla \nu \nabla \phi d x
$$

and

$$
\int_{\mathbb{R}^{N}} V(x) v_{n} \phi d x \rightarrow \int_{\mathbb{R}^{N}} V(x) v \phi d x
$$

and

$$
\int_{\mathbb{R}^{N}}\left[|x|^{-\mu} *\left|v_{n}\right|^{p}\right]\left|v_{n}\right|^{p-2} v_{n} \phi d x \rightarrow \int_{\mathbb{R}^{N}}\left[|x|^{-\mu} *|v|^{p}\right]|v|^{p-2} v \phi d x .
$$

Consequently, according to the above facts, we can obtain

$$
0=\int_{\mathbb{R}^{N}} \nabla v \nabla \phi d x+\int_{\mathbb{R}^{N}} V(x) v \phi d x-\int_{\mathbb{R}^{N}}\left[|x|^{-\mu} *|v|^{p}\right]|v|^{p-2} v \phi d x
$$

for all $\phi \in C_{0}^{\infty}\left(\mathbb{R}^{N}\right)$. Since $C_{0}^{\infty}\left(\mathbb{R}^{N}\right)$ is dense in $E$, for any $\phi \in E$, there exists a sequence $\left\{\phi_{n}\right\} \subset C_{0}^{\infty}\left(\mathbb{R}^{N}\right)$ such that $\phi_{n} \rightarrow \phi$ in $E$. Thereby,

$$
0=\int_{\mathbb{R}^{N}} \nabla v \nabla \phi_{n} d x+\int_{\mathbb{R}^{N}} V(x) v \phi_{n} d x-\int_{\mathbb{R}^{N}}\left[|x|^{-\mu} *|v|^{p}\right]|v|^{p-2} v \phi_{n} d x .
$$

Let $n \rightarrow \infty$, by the Hardy-Littlewood-Sobolev inequality, Hölder's inequality, and the Sobolev embedding theorem, we get

$$
0=\int_{\mathbb{R}^{N}} \nabla v \nabla \phi d x+\int_{\mathbb{R}^{N}} V(x) v \phi d x-\int_{\mathbb{R}^{N}}\left[|x|^{-\mu} *|v|^{p}\right]|v|^{p-2} v \phi d x,
$$

which yields that $J^{\prime}(v)=0$. Notice that

$$
\begin{aligned}
o(1) & =\left\langle J^{\prime}\left(v_{n}\right)-J^{\prime}(v), v_{n}-v\right\rangle \\
& =\left\|v_{n}-v\right\|^{2}-\int_{\mathbb{R}^{N}}\left(\left[|x|^{-\mu} *\left|v_{n}\right|^{p}\right]\left|v_{n}\right|^{p-2} v_{n}-\left[|x|^{-\mu} *|v|^{p}\right]|v|^{p-2} v\right)\left(v_{n}-v\right) d x .
\end{aligned}
$$


As in the proof of Lemma 2.10, we know that there is a constant $c_{3}>0$ such that

$$
\left\|v_{n}-v\right\|^{2} \leq c_{3}\left\|v_{n}-v\right\|_{2 N p}^{2}{ }_{2 N-\mu}+o(1)
$$

If $\left\|v_{n}-v\right\|_{\frac{2 N p}{2 N-\mu}} \nrightarrow 0$ as $n \rightarrow \infty$, then by the boundedness of $\left\{v_{n}\right\}$ in $E$ and the vanishing lemma [38, Lemma 1.21] we have

$$
\sup _{y \in \mathbb{R}^{N}} \int_{B_{1}(y)}\left|v_{n}-v\right|^{\frac{2 N p}{2 N-\mu}} d x \nrightarrow 0
$$

Consequently, up to a subsequence, there exists $\varepsilon_{0}>0$ such that

$$
\sup _{y \in \mathbb{R}^{N}} \int_{B_{1}(y)}\left|v_{n}-v\right|^{\frac{2 N p}{2 N-\mu}} d x \geq \varepsilon_{0}, \quad \forall n \in \mathbb{N} \text {. }
$$

The proof of Lemma 2.5 indicates that there exists $y_{n} \in \mathbb{R}^{N}$ such that

$$
\int_{B_{1}\left(y_{n}\right)}\left|v_{n}-v\right|^{\frac{2 N p}{2 N-\mu}} d x=\sup _{y \in \mathbb{R}^{N}} \int_{B_{1}(y)}\left|v_{n}-v\right|^{\frac{2 N p}{2 N-\mu}} d x \geq \varepsilon_{0}, \quad \forall n \in \mathbb{N} .
$$

By the assumption of periodicity, we can assume that $\left\{y_{n}\right\}$ is bounded in $\mathbb{R}^{N}$. Therefore, there exists a bounded domain $\Omega_{1} \subset \mathbb{R}^{N}$ such that

$$
\int_{\Omega_{1}}\left|v_{n}-v\right|^{\frac{2 N p}{2 N-\mu}} d x \geq \int_{B_{1}\left(y_{n}\right)}\left|v_{n}-v\right|^{\frac{2 N p}{2 N-\mu}} d x \geq \varepsilon_{0} .
$$

However, since $\Omega_{1}$ is bounded, the embedding theorem implies that

$$
\int_{\Omega_{1}}\left|v_{n}-v\right|^{\frac{2 N p}{2 N-\mu}} d x \rightarrow 0
$$

Clearly, this yields a contraction. Hence $\left\|v_{n}-v\right\|_{\frac{2 N p}{2 N-\mu}} \rightarrow 0$ as $n \rightarrow \infty$. Then, by (2.2), we see that $v_{n} \rightarrow v$ in $E$ and $J(v)=c>0$. Therefore, $v \in \mathcal{N}$ is a ground state solution of problem (1.1). The proof is completed.

Next we are devoted to looking for infinitely many geometrically distinct solutions for problem (1.1). Observe that, by Lemma 2.8-(iv), we know that $\psi$ is even. Set

$$
K:=\left\{v \in S: \psi^{\prime}(v)=0\right\} \quad \text { and } \quad K_{c}:=\{v \in K: \psi(v)=c\}
$$

and

$$
U_{\delta}\left(K_{c}\right):=\left\{w \in S: d\left(w, K_{c}\right)<\delta\right\} .
$$

Let

$$
\Sigma:=\{A \subset S: \bar{A}=A,-A=A\} .
$$


For $A \in \Sigma$, we denote the Krasnoselskii genus of $A$ by $i(A)$. Define

$$
c_{k}:=\inf \left\{d \in \mathbb{R}: d \geq c, i\left(\psi^{d}\right) \geq k\right\}
$$

for all $k \in \mathbb{N}$. It is not difficult to prove that $c \leq c_{k}$ and $c_{k} \leq c_{k+1}$.

Lemma $2.12 c_{k}$ is a critical value of $\psi$.

Proof If the conclusion is false, then for any $w \in S$ one has $\psi(w) \neq c_{k}$ or $\psi^{\prime}(w) \neq 0$. It implies that there exists $\delta>0$ such that

$$
N_{c_{k}, \delta}:=\left\{w \in S:\left|\psi(w)-c_{k}\right|<\delta,\left\|\psi^{\prime}(w)\right\|_{E^{*}}<\delta\right\}=\emptyset .
$$

Otherwise, there exists a sequence $\left\{w_{n}\right\} \subset S$ such that $\psi\left(w_{n}\right) \rightarrow c_{k}$ and $\left\|\psi^{\prime}\left(w_{n}\right)\right\|_{E^{*}} \rightarrow 0$ as $n \rightarrow \infty$. Set $v_{n}=m\left(w_{n}\right)$, then Lemma 2.8-(ii) shows that $\left\{v_{n}\right\} \subset \mathcal{N}$ is a $(P S)_{c_{k}}$ sequence of $J$. Moreover, $\left\{v_{n}\right\}$ is bounded in $E$ by Lemma 2.5. Hence, up to a subsequence, there exists $v \in E$ such that $v_{n} \rightarrow v$ in $E, v_{n} \rightarrow v$ in $L_{l o c}^{q}\left(\mathbb{R}^{N}\right)$ for any $2 \leq q<2^{*}$, and $v_{n}(x) \rightarrow v(x)$ a.e. on $\mathbb{R}^{N}$. With a similar argument as the proof of Lemma 2.11, we can deduce that $J^{\prime}(v)=0$ and $v_{n} \rightarrow v$ in $E$. Therefore, $J(v)=c_{k} \geq c>0$ and $v \in \mathcal{N}$. Lemma 2.8-(iii) implies that $w:=m^{-1}(v) \in K_{c_{k}}$, a contradiction. As a consequence, $N_{c_{k}, \delta}=\emptyset$. By using a deformation lemma (see Theorem 3.4 in [35]), there exists $\varepsilon_{0}>0$ such that, for any $0<\varepsilon<\bar{\varepsilon} \leq \varepsilon_{0}$, there exists a continuous 1-parameter family of homeomorphisms $\eta(t, \cdot)$ of $S, 0 \leq t<\infty$, admitting the properties:

(i) $\eta(w, t)=w$ if $t=0$ or $\psi^{\prime}(w)=0$, or $\left|\psi(w)-c_{k}\right| \geq \bar{\varepsilon}$;

(ii) $\psi(\eta(w, t))$ is nonincreasing in $t$ for any $w \in S$;

(iii) $\eta\left(\psi^{c_{k}+\varepsilon}, 1\right) \subset \psi^{c_{k}-\varepsilon}$;

(iv) $\eta(\cdot, s) \circ \eta(\cdot, t)=\eta(\cdot, s+t)$ for all $s, t \geq 0$;

(v) $\eta(w, t)$ is odd in $w$ for $t \geq 0$.

Since $N_{c_{k}, \delta}=\emptyset$, then there exists $0<\varepsilon_{1}<\varepsilon_{0}$ such that

$$
\psi_{c_{k}-\varepsilon_{1}}^{c_{k}+\varepsilon_{1}} \cap K=\emptyset
$$

For each $w \in \psi^{c_{k}+\varepsilon_{1}}$, using deformation shrinkage property (iii), we get $\psi(\eta(w, 1)) \leq c_{k}-\varepsilon_{1}$. Let $\tau=\tau(w)$ be the infimum of the time for which $\psi(\eta(w, t)) \leq c_{k}-\varepsilon_{1}$. We can prove that $\tau: \psi^{c_{k}+\varepsilon_{1}} \rightarrow[0,+\infty)$ is a continuous mapping, and since $\psi$ is even, then $\tau(-w)=\tau(w)$. For $w \in \psi^{c_{k}+\varepsilon_{1}}$, set $h(w):=\eta(w, \tau(w))$. Then $h$ is odd and continuous. Making use of the mapping property of the genus and the definition of $c_{k}$, we can conclude that

$$
k \leq i\left(\psi^{c_{k}+\varepsilon_{1}}\right) \leq i\left(\psi^{c_{k}-\varepsilon_{1}}\right) \leq k-1
$$

which yields a contradiction. The proof is completed.

Set

$$
\tilde{K}:=\bigcup_{k=1}^{\infty} K_{c_{k}} .
$$


Choose $\mathscr{F} \subset \tilde{K}$ such that $\mathscr{F}=-\mathscr{F}$ and each orbit $\mathcal{O}(w) \subset K$ has a unique representative in $\mathscr{F}$. Following the proof of Lemma 2.11 in [40] and some standard arguments, it is easy to prove that $\mathscr{F}$ is an infinite set. As a result, $\psi$ admits infinitely many pairs of geometrically distinct critical points. Consequently, using Lemma 2.8-(iii) and Lemma 2.11, we can see that problem (1.1) has infinitely many pairs of geometrically distinct solutions. The proof of Theorem 1.1 is completed.

\section{Acknowledgements}

We would like to thank the referees for their valuable comments and suggestions. The second author would like to thank the China Scholarship Council and the Embassy of the People's Republic of China in Romania.

\section{Funding}

This work was supported by the Natural Science Fund of Hunan Province (2021 JJ30189), the Scientific Research Fund of Hunan Provincial Education Department (18B342, 19C1700) and the China Scholarship Council (201908430219).

\section{Availability of data and materials}

Not applicable.

\section{Declarations}

\section{Competing interests}

The authors declare that they have no competing interests.

\section{Authors' contributions}

TW and WZ conceived of the idea of this manuscript and wrote the manuscript. Both authors contributed equally to the writing of this paper, and both authors read and approved the final manuscript.

\section{Author details}

${ }^{1}$ School of Educational Sciences, Baotou Teachers College, Baotou, 014030, Inner Mongolia, P.R. China. ${ }^{2}$ College of Science, Hunan University of Technology and Business, Changsha, 410205, Hunan, P.R. China. ${ }^{3}$ Key Laboratory of Hunan Province for Statistical Learning and Intelligent Computation, Hunan University of Technology and Business, Changsha, 410205, Hunan, P.R. China. ${ }^{4}$ Department of Mathematics, University of Craiova, Street A.I. Cuza no. 13, 200585, Craiova, Romania.

\section{Publisher's Note}

Springer Nature remains neutral with regard to jurisdictional claims in published maps and institutional affiliations.

Received: 23 September 2021 Accepted: 29 October 2021 Published online: 19 November 2021

\section{References}

1. Alves, C.O., Nóbrega, A.B., Yang, M.: Multi-bump solutions for Choquard equation with deepening potential well. Calc. Var. Partial Differ. Equ. 55, 1-28 (2016)

2. Bergé, L., Couairon, A.: Nonlinear propagation of self-guided ultra-short pulses in ionized gases. Phys. Plasmas 7, 210-230 (2000)

3. Chen, S., Wu, X.: Existence of positive solutions for a class of quasilinear Schrödinger equations of Choquard type. J. Math. Anal. Appl. 475, 1754-1777 (2019)

4. Chen, W., Li, C., Ou, B.: Classification of solutions for an integral equation. Commun. Pure Appl. Math. 59, 330-343 (2006)

5. Cingolani, S., Clapp, M., Secchi, S.: Multiple solutions to a magnetic nonlinear Choquard equation. Z. Angew. Math. Phys. 63, 233-248 (2012)

6. Cingolani, S., Secchi, S., Squassina, M.: Semi-classical limit for Schrödinger equations with magnetic field and Hartree-type nonlinearities. Proc. R. Soc. Edinb. A 140, 973-1009 (2010)

7. Clapp, M., Salazar, D.: Positive and sign changing solutions to a nonlinear Choquard equation. J. Math. Anal. Appl. 407, $1-15(2013)$

8. d'Avenia, P., Siciliano, G., Squassina, M.: On fractional Choquard equations. Math. Models Methods Appl. Sci. 25 1447-1476 (2015)

9. Dalfovo, F., Giorgini, S., Pitaevskii, L.P., Stringari, S.: Theory of Bose-Einstein condensation in trapped gases. Rev. Mod. Phys. 71, 463-512 (1999)

10. Ding, Y., Gao, F., Yang, M.: Semiclassical states for Choquard type equations with critical growth: critical frequency case. Nonlinearity 33,6695-6728 (2020)

11. Ekeland, I.: On the variational principle. J. Math. Anal. Appl. 47, 324-353 (1974)

12. Fang, X., Szulkin, A.: Multiple solutions for a quasilinear Schrödinger equation. J. Differ. Equ. 254, 2015-2032 (2013)

13. Gao, F., Rădulescu, V.D., Yang, M., Zheng, Y.: Standing waves for the pseudo-relativistic Hartree equation with Berestycki-Lions nonlinearity. J. Differ. Equ. 295, 70-112 (2021)

14. Gao, F., Yang, M.: A strongly indefinite Choquard equation with critical exponent due to the Hardy-Littlewood-Sobolev inequality. Commun. Contemp. Math. 20, 1750037 (2018) 
15. Gao, F., Yang, M., Santos, C.A., Zhou, J.: Infinitely many solutions for a class of critical Choquard equation. Topol. Methods Nonlinear Anal. 54, 219-232 (2019)

16. Ghimenti, M., Van Schaftingen, J.: Nodal solutions for the Choquard equation. J. Funct. Anal. 271, 107-135 (2016)

17. Li, C., Ma, L.: Uniqueness of positive bound states to Schrödinger systems with critical exponents. SIAM J. Math. Anal. 40, 1049-1057 (2008)

18. Lieb, E.H.: Existence and uniqueness of the minimizing solution of Choquard' nonlinear equation. Stud. Appl. Math. 57, 93-105 (1977)

19. Lieb, E.H., Loss, M.: Analysis, 2nd edn. Grad. Stud. Math., vol. 14. Am. Math. Soc., Providence (2001)

20. Lions, P.L.: The Choquard equation and related questions. Nonlinear Anal. 4, 1063-1072 (1980)

21. Litvak, A.G.: Self-focusing of powerful light beams by thermal effects. JETP Lett. 4, 230-232 (1966)

22. Ma, L., Zhao, L.: Classification of positive solitary solutions of the nonlinear Choquard equation. Arch. Ration. Mech. Anal. 195, 455-467 (2010)

23. Ma, P., Zhang, J.: Existence and multiplicity of solutions for fractional Choquard equations. Nonlinear Anal. 164, 100-117 (2017)

24. Moroz, V., Van Schaftingen, J.: Groundstates of nonlinear Choquard equations: existence, qualitative properties and decay asymptotics. J. Funct. Anal. 265, 153-184 (2013)

25. Moroz, V., Van Schaftingen, J.: Semi-classical states for the Choquard equation. Calc. Var. Partial Differ. Equ. 52 199-235 (2015)

26. Moroz, V., Van Schaftingen, J.: Existence of groundstates for a class of nonlinear Choquard equations. Trans. Am. Math. Soc. 367, 6557-6579 (2015)

27. Moroz, V., Van Schaftingen, J.: Groundstates of nonlinear Choquard equations: Hardy-Littlewood-Sobolev critical exponent. Commun. Contemp. Math. 17, 1550005 (2015)

28. Pekar, S.: Untersuchungen über die Elektronentheorie der Kristalle. Akademie Verlag, Berlin (1954)

29. Penrose, R.: On gravity's role in quantum state reduction. Gen. Relativ. Gravit. 28, 581-600 (1996)

30. Qin, D., Lai, L., Yuan, S., Wu, Q:: Ground states and multiple solutions for Choquard-Pekar equations with indefinite potential and general nonlinearity. J. Math. Anal. Appl. 500, 125143 (2021)

31. Qin, D., Rădulescu, V.D., Tang, X.: Ground states and geometrically distinct solutions for periodic Choquard-Pekar equations. J. Differ. Equ. 275, 652-683 (2021)

32. Qin, D., Tang, X.: On the planar Choquard equation with indefinite potential and critical exponential growth. J. Differ Equ. 285, 40-98 (2021)

33. Secchi, S.: A note on Schrödinger-Newton systems with decaying electric potential. Nonlinear Anal. 72, 3842-3856 (2010)

34. Shen, Z., Gao, F., Yang, M.: Ground states for nonlinear fractional Choquard equations with general nonlinearities. Math. Methods Appl. Sci. 39, 4082-4098 (2015)

35. Struwe, M.: Variational Methods, 2nd edn. Springer, Berlin (1996)

36. Szulkin, A., Weth, T.: The method of Nehari manifold. In: Gao, D.Y., Motreanu, D. (eds.) Handbook of Nonconvex Analysis and Applications, pp. 597-632. International Press, Boston (2010)

37. Wei, J., Winter, M.: Strongly interacting bumps for the Schrödinger-Newton equations. J. Math. Phys. 50, 012905 (2009)

38. Willem, M.: Minimax Theorem. Birkhäuser, Boston (1996)

39. Wu, Q., Qin, D., Chen, J.: Ground states and non-existence results for Choquard type equations with lower critical exponent and indefinite potentials. Nonlinear Anal. 197, 111863 (2020)

40. Wu, X., Wu, K.: Geometrically distinct solutions for quasilinear elliptic equations. Nonlinearity 27, 987-1001 (2014)

41. Xiang, M., Rădulescu, V.D., Zhang, B.: A critical fractional Choquard-Kirchhoff problem with magnetic field. Commun. Contemp. Math. 21, 1850004 (2019)

42. Zhang, J., Wu, Q., Qin, D.: Semiclassical solutions for Choquard equations with Berestycki-Lions type conditions. Nonlinear Anal. 188, 22-49 (2019)

43. Zhang, J., Zhang, W., Tang, X.: Ground state solutions for Hamiltonian elliptic system with inverse square potential. Discrete Contin. Dyn. Syst. 37, 4565-4583 (2017)

44. Zhang, J., Zhang, W., Xie, X.: Infinitely many solutions for a gauged nonlinear Schrödinger equation. Appl. Math. Lett. $88,21-27$ (2019)

\section{Submit your manuscript to a SpringerOpen ${ }^{\circ}$ journal and benefit from:}

- Convenient online submission

- Rigorous peer review

- Open access: articles freely available online

- High visibility within the field

- Retaining the copyright to your article

Submit your next manuscript at $\gg$ springeropen.com 